\title{
Avaliação do SISVAN na gestão de ações de alimentação e nutrição em Minas Gerais, Brasil
}

\author{
Evaluation of the Food and Nutrition Surveillance System \\ (SISVAN) in food and nutritional management services \\ in the State of Minas Gerais, Brazil
}

\author{
Mara Diana Rolim ${ }^{1}$ \\ Sheyla Maria Lemos Lima ${ }^{1}$ \\ Denise Cavalcante de Barros ${ }^{2}$ \\ Carla Lourenço Tavares de Andrade ${ }^{1}$
}

${ }^{1}$ Departamento de Administração e

Planejamento em Saúde, Escola Nacional de Saúde Pública Sérgio Arouca (Ensp), Fundação Oswaldo Cruz (Fiocruz). R. Leopoldo Bulhões 1480, Manguinhos. 21041-210 Rio de Janeiro RJ Brasil. mararolim@yahoo.com.br ${ }^{2}$ Centro de Saúde Escola Germano Sinval Faria, Ensp, Fiocruz.

\begin{abstract}
The scope of this article is to evaluate the SISVAN as a tool for planning, management and evaluation of food and nutrition actions in primary healthcare in the Unified Health System (SUS). It involved a cross-sectional study composed of a stratified random sample of the municipalities in the State of Minas Gerais. The subjects of the research were municipal officials of SISVAN who filled out a structured questionnaire. Descriptive analysis of the data was performed with the construction of simple and bivariate tables. It was observed that those responsible for SISVAN, collect (50\%) and input (55\%) weight, height, and food consumption data; whereas 53\%, 59\% and $71 \%$ do not analyze and do not recommend or perform nutrition actions, respectively. This being the case, most of those responsible do not use the information for planning, management and evaluation of food and nutrition traits. The findings show that the SISVAN is not used to its full potential; the data generated have not been used for planning, management and evaluation of nutrition services in primary healthcare in the SUS. Key words Nutritional surveillance, Nutrition programs, Primary Healthcare, Unified Health System, Health management
\end{abstract}

Resumo O objetivo deste artigo é avaliar o SISVAN como instrumento para o planejamento, gestão e avaliação de ações de alimentação e nutrição na atenção básica do SUS. Estudo transversal composto por uma amostra aleatória estratificada dos municípios do estado de Minas Gerais. Os sujeitos da pesquisa foram os responsáveis municipais pelo SISVAN, que responderam a um questionário estruturado. A análise descritiva dos dados foi realizada com a construção de tabelas simples e bivariadas. Observou-se que os responsáveis pelo SISVAN coletam (50\%) e digitam (55\%) dados de peso, altura e consumo alimentar, enquanto que 53\%, 59\% e 71\% não os analisam, não recomendam e nem executam ações de nutrição, respectivamente. Sendo assim, a maior parte dos responsáveis não utiliza as informações para o planejamento, a gestão e a avaliação da alimentação e nutrição. Os achados evidenciam que o SISVAN não é utilizado em seu potencial; as informações geradas não têm sido empregadas para o planejamento, a gestão e a avaliação dos serviços de nutrição na atenção básica no SUS.

Palavras-chave Vigilância nutricional, Programas de nutrição, Atenção primária à saúde, Sistema único de saúde, Gestão em saúde 


\section{Introdução}

No Brasil, desde a década de 70, observou-se uma transição nutricional, com o aumento da prevalência do sobrepeso e obesidade na população e diminuição da desnutrição, especialmente entre as crianças menores de 5 anos $^{1-3}$, associada ao aumento das doenças crônicas não transmissíveis (DCNT), as quais foram responsáveis por $72 \%$ das mortes em 2007. Os hábitos alimentares neste contexto são considerados importantes fatores de risco a serem monitorados ${ }^{4}$. A análise dos perfis nutricionais e alimentares aponta para a identificação de grupos de risco que, aliada às especificidades locais de cada contexto ${ }^{5-7}$, contribuem para um adequado planejamento e implementação de ações de promoção da saúde, prevenção e cuidado.

Desde 1990, o Ministério da Saúde (MS) adota um sistema de informações para o monitoramento das condições nutricionais e alimentares no âmbito da atenção básica do Sistema Único de Saúde (SUS). O Sistema de Vigilância Alimentar e Nutricional (SISVAN) permite gerenciar informações de todas as fases da vida e tem como destaque populações tradicionais e grupos sob vulnerabilidade social ${ }^{3,8}$. A estratégia compõe uma das diretrizes da Política Nacional de Alimentação e Nutrição (PNAN), constituindo-se em importante ferramenta para a tomada de decisão, implementação de ações, avaliação e adequação nos diferentes níveis de gestão da atenção à saúde ${ }^{8-11}$.

De maneira geral, os Sistemas de Informação em Saúde (SIS) têm sido subutilizados em seu potencial de instrumentalizar a tomada de decisão pelos gestores ${ }^{12}$. O mesmo é observado em relação ao SISVAN ${ }^{13-15}$. Em 2006, um estudo realizado junto aos estados e capitais no país evidenciou que, apesar dos avanços geográficos da implantação do SISVAN nos municípios brasileiros, o sistema não era utilizado em todo seu potencial, como esperado pelo governo federal ${ }^{13}$.

A adequada utilização do referido sistema contribuiria para melhoria da qualidade da coleta, do registro dos dados e confiabilidade das informações geradas aprimorando consequentemente o planejamento e implementação das ações propostas pela PNAN nas diferentes esferas de governo. Um outro potencial do sistema é que as informações geradas são continuas e úteis para o uso imediato - informação para a ação - tanto para os gestores, no planejamento dos planos de ação, como para os profissionais, na reorganização atendimento nutricional na unidade de saúde ${ }^{11}$.

O objetivo deste estudo foi avaliar o uso do SISVAN como instrumento de informação para o planejamento, gestão e avaliação de ações de alimentação e nutrição no âmbito da atenção básica do SUS nos municípios de Minas Gerais, tendo em vista contribuir para a revisão das estratégias e ações de implementação da PNAN.

O trabalho de justifica pela escassez de pesquisas avaliativas no país sobre o uso do SISVAN pelos tomadores de decisão em quaisquer instâncias governamentais. Assim, as questões reveladas pelo presente estudo poderiam ser úteis para outras regiões do país, uma vez que, de um modo geral, o estado de Minas Gerais apresenta características similares quanto à distribuição populacional, densidade demográfica e aspectos socioeconômicos.

\section{Métodos}

Trata-se de um estudo transversal e descritivo, desenvolvido no âmbito da atenção básica do SUS, nos municípios de Minas Gerais, no ano de 2012.

Considerando que Minas Gerais possui 853 municípios, foi selecionada uma amostra representativa, aleatória e estratificada de 268 municípios, composta por 12 estratos (Tabela 1), de acordo com os seguintes critérios:

a) porte populacional (até 10.000; de 10.001 a 80.000 e mais do que 80.000 habitantes), adaptado do censo demográfico de $2010^{16}$;

b) cobertura do SISVAN (número de crianças menores de 10 anos registradas no SISVAN ${ }^{17}$ no índice Peso/Idade dividido pelo número de crianças menores de 10 anos registradas no Sistema de Informação da Atenção Básica $(\mathrm{SIAB})^{10}$, em 2010, em Minas Gerais);

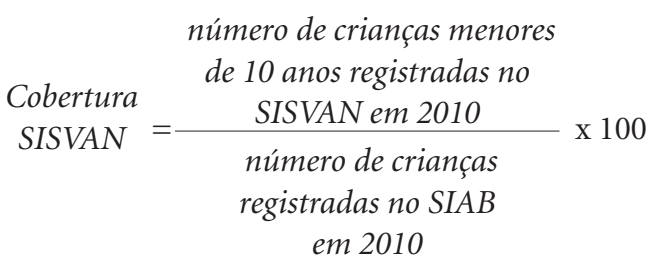

c) cobertura do SIAB (número de crianças menores de 10 anos registradas no SIAB dividido pelo número de crianças menores de 10 anos identificadas pelo Censo Demográfico, 2010 ${ }^{16}$ ). 


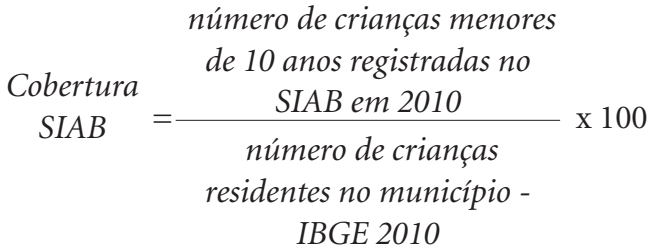

Considerando a inexistência de um valor padrão para análise das coberturas, adotou-se a mediana como ponto de corte: $37 \%$ de cobertura do SISVAN e $88 \%$ de cobertura do SIAB. Os municípios (2\%) que não apresentaram dados sobre o SIAB foram excluídos da população.

Para o cálculo do tamanho da amostra (corrigido para população finita) levou-se em consideração os parâmetros: intervalo de confiança $(z)$ de $95 \%$, proporção de não resposta do SISVAN pelos municípios $(p)$ de $50 \%$ e erro tolerável $(e)$ de amostragem de $5 \%{ }^{18}$, conforme apresentado na fórmula a seguir:

$$
\mathrm{n}=\frac{z^{2} \times \hat{\mathrm{p}} \mathrm{x} \hat{\mathrm{q}}}{e^{2}} \times \sqrt{\frac{N-n}{N-1}}
$$

Após a consolidação dos resultados, atribuiuse peso à amostra considerando o inverso da fração de amostragem $(\mathrm{n} / \mathrm{N})$, e os resultados do total de municípios do estado foram apresentados de forma expandida.

Os sujeitos da pesquisa foram os responsáveis municipais, formais ou não, pelo SISVAN, identificados pelas Regionais de Saúde ou por contato direto com as Secretarias Municipais de Saúde.
Os dados primários foram coletados por meio de questionário estruturado autoaplicado em formato eletrônico considerando a viabilidade financeira, o tempo disponível e a distância territorial. $\mathrm{O}$ questionário foi construído com base em questões levantadas em reuniões com as Regionais de Saúde prévias à pesquisa; em outros questionários de estudos de avaliação do SISVAN $^{13,19}$ e nas diretrizes da PNAN ${ }^{3,7}$ abordou-se questões referentes ao perfil do responsável pelo SISVAN, à coordenação da gestão do SISVAN e à utilização de suas informações.

O primeiro contato foi realizado com os secretários municipais de saúde por meio de correspondência e, posteriormente, com os responsáveis pelo SISVAN por correspondência, telefone e e-mail.

Todos os contatos foram feitos pela pesquisadora e uma estagiária. A coleta de dados ocorreu entre 23 de abril e 21 de julho de 2012. A análise descritiva dos dados foi feita no software estatístico Statistical Package for Social Sciences (SPSS) versão 17.0 , por meio da construção de tabelas simples e bivariadas.

Após a consolidação dos resultados, atribuiu-se peso à amostra considerando o inverso da fração de amostragem $(\mathrm{n} / \mathrm{N})$, e os resultados foram apresentados de forma expandida para a população. A participação dos entrevistados foi voluntária, firmada por meio de um Termo de Consentimento Livre e Esclarecido, respeitandose as premissas da resolução 196/96 do Conselho Nacional de Saúde ${ }^{20}$ e da Comissão de Ética em Pesquisa (CEP) da ENSP/Fiocruz.

Tabela 1. Frequência e porcentagem da população e da amostra em cada estrato - Minas Gerais, 2012.

\begin{tabular}{clccrrr}
\hline Estratos & \multicolumn{1}{c}{$\begin{array}{c}\text { População } \\
\text { do Município }\end{array}$} & $\begin{array}{c}\text { Cobertura } \\
\text { do SISVAN }\end{array}$ & $\begin{array}{c}\text { Cobertura } \\
\text { do SIAB }\end{array}$ & $\begin{array}{c}\text { População } \\
\text { N }\end{array}$ & \multicolumn{2}{c}{$\begin{array}{c}\text { Amostra } \\
\text { N }\end{array}$} \\
\hline 1 & Até 10.000 habitantes & $<37 \%$ & $<88 \%$ & 53 & 6,3 & 17 \\
2 & Até 10.000 habitantes & $\geq 37 \%$ & $<88 \%$ & 121 & 14,5 & 39 \\
3 & Até 10.000 habitantes & $<37 \%$ & $\geq 88 \%$ & 167 & 20,0 & 53 \\
4 & Até 10.000 habitantes & $\geq 37 \%$ & $\geq 88 \%$ & 141 & 16,9 & 45 \\
5 & Entre 10.001 e 80.000 habitantes & $<37 \%$ & $<88 \%$ & 94 & 11,2 & 30 \\
6 & Entre 10.001 e 80.000 habitantes & $\geq 37 \%$ & $<88 \%$ & 103 & 12,3 & 33 \\
7 & Entre 10.001 e 80.000 habitantes & $<37 \%$ & $\geq 88 \%$ & 75 & 9,0 & 24 \\
8 & Entre 10.001 e 80.000 habitantes & $\geq 37 \%$ & $\geq 88 \%$ & 41 & 4,9 & 13 \\
9 & Mais de 80.000 habitantes & $<37 \%$ & $<88 \%$ & 27 & 3,2 & 9 \\
10 & Mais de 80.000 habitantes & $\geq 37 \%$ & $<88 \%$ & 12 & 1,4 & 4 \\
11 & Mais de 80.000 habitantes & $<37 \%$ & $\geq 88 \%$ & 2 & 0,2 & 1 \\
12 & Mais de 80.000 habitantes & $\geq 37 \%$ & $\geq 88 \%$ & - & - & - \\
& Total & & & $\mathbf{8 3 6}$ & $\mathbf{1 0 0 , 0}$ & $\mathbf{2 6 8}$
\end{tabular}

"A população não finaliza em 853 municípios porque 17 foram excluídos por não constarem dados do SIAB, em 2010. 


\section{Resultados}

A grande maioria dos responsáveis pelo SISVAN é do sexo feminino, menor que 39 anos, com nível superior e titulação mínima de especialização (finalizada ou em andamento). Predominam profissionais da área da saúde, em especial enfermeiros $(36,6 \%)$ e nutricionistas $(38,8 \%)$. Mais da metade não tem vínculo empregatício efetivo e trabalham há dois anos ou menos com o SISVAN. A quase totalidade trabalha com o SISVAN e com outras ações (97,0\%) (Tabela 2), dentre elas, coordenação das ações de alimentação e nu- trição, condicionalidades da saúde no Programa Bolsa Família (PBF) e Programa Nacional de Suplementação de Micronutrientes.

Observa-se que $51,8 \%$ dos responsáveis pelo SISVAN participaram de uma capacitação ocorrida, em 73,2\% dos casos, a menos de três anos e que em sua maioria (95\%) foi organizada pelo nível estadual.

Dos entrevistados, $65,4 \%$ informaram que a coordenação do SISVAN encontra-se formalmente vinculada à atenção básica e, $24,4 \%$, às vigilâncias (epidemiológica e em saúde). Observa-se que $83,3 \%$ referiram parceria com a aten-

Tabela 2. Distribuição dos responsáveis $(\mathrm{N}=836)$ pelo SISVAN por sexo, idade, escolaridade, profissão, vínculo empregatício, tempo de trabalho e trabalho exclusivo com o SISVAN - Minas Gerais, 2012.

\begin{tabular}{|c|c|c|c|}
\hline Variável & $\mathbf{N}$ & $\%$ & IC 95\% \\
\hline \multicolumn{4}{|l|}{ Sexo } \\
\hline Feminino & 717 & 85,8 & 81,$0 ; 89,5$ \\
\hline Masculino & 116 & 13,9 & 10,$2 ; 18,6$ \\
\hline Sem resposta & 3 & 0,4 & 0,$1 ; 2,6$ \\
\hline \multicolumn{4}{|l|}{ Idade (anos) } \\
\hline$<30$ & 431 & 51,6 & 45,$5 ; 57,5$ \\
\hline 30 a 39 & 268 & 32,1 & 26,$7 ; 38,0$ \\
\hline$>40$ & 136 & 16,3 & 12,$3 ; 21,3$ \\
\hline \multicolumn{4}{|l|}{ Escolaridade $^{\mathrm{a}}$} \\
\hline Nível médio incompleto & 3 & 0,4 & 0,$1 ; 2,6$ \\
\hline Nível médio completo/Superior incompleto & 166 & 19,8 & 15,$5 ; 25,1$ \\
\hline Superior completo & 272 & 32,6 & 27,$2 ; 38,5$ \\
\hline Especialização finalizada ou em andamento & 364 & 43,6 & 37,$7 ; 49,7$ \\
\hline Mestrado finalizado ou em andamento & 28 & 3,3 & 1,$8 ; 6,0$ \\
\hline Sem resposta & 3 & 0,4 & 0,$1 ; 2,6$ \\
\hline \multicolumn{4}{|l|}{ Profissão ${ }^{\mathrm{b}}$} \\
\hline Técnico de Enfermagem & 38 & 4,5 & 2,$6 ; 7,7$ \\
\hline Técnico de Informática & 25 & 3,0 & 1,$5 ; 5,9$ \\
\hline Enfermeiro & 306 & 36,6 & 31,$0 ; 42,6$ \\
\hline Nutricionista & 283 & 33,8 & 28,$5 ; 39,6$ \\
\hline Médico & 3 & 0,4 & 0,$1 ; 2,7$ \\
\hline Outra profissão & 178 & 21,3 & 16,$8 ; 26,7$ \\
\hline Sem resposta & 3 & 0,4 & 0,$1 ; 2,6$ \\
\hline \multicolumn{4}{|l|}{ Vínculo empregatício } \\
\hline Efetivo & 364 & 43,6 & 37,$7 ; 49,6$ \\
\hline Contratado/Prestador & 463 & 55,3 & 49,$3 ; 61,2$ \\
\hline Sem resposta & 9 & 1,1 & 0,$4 ; 3,5$ \\
\hline \multicolumn{4}{|l|}{ Tempo de trabalho com o SISVAN } \\
\hline Menos de 1 ano & 247 & 29,5 & 24,$3 ; 35,4$ \\
\hline Entre 1 e 2 anos & 234 & 28,0 & 22,$9 ; 33,8$ \\
\hline Entre 3 e 4 anos & 197 & 23,5 & 18,$8 ; 29,0$ \\
\hline 5 anos ou mais & 142 & 17,0 & 13,$0 ; 22,0$ \\
\hline Sem Resposta & 16 & 1,9 & 0,$8 ; 4,4$ \\
\hline \multicolumn{4}{|l|}{ Trabalha com outras ações além do SISVAN } \\
\hline Não & 19 & 2,2 & 1,$0 ; 4,9$ \\
\hline Sim & 811 & 97,0 & 94,$1 ; 98,5$ \\
\hline Não sabe informar & 6 & 0,7 & 0,$2 ; 3,0$ \\
\hline
\end{tabular}

${ }^{a}$ Nenhum responsável pelo SISVAN possui doutorado. ${ }^{b}$ Nenhum responsável pelo SISVAN é assistente social. 
Tabela 3. Distribuição dos responsáveis $(\mathrm{N}=836)$ pelo SISVAN por atividade executada - Minas Gerais, 2012.

\begin{tabular}{|c|c|c|c|c|c|c|}
\hline \multirow{2}{*}{ Atividades do SISVAN } & \multicolumn{3}{|c|}{ Executadas } & \multicolumn{3}{|c|}{ Não executadas } \\
\hline & $\mathbf{N}$ & $\%$ & IC 95\% & $\mathbf{N}$ & $\%$ & IC 95\% \\
\hline Coleta de dados ${ }^{\mathrm{a}}$ & 422 & 50,5 & 44,$6 ; 56,4$ & 414 & 49,5 & 43,$6 ; 55,4$ \\
\hline Digitação dos dados no SISVAN Web & 462 & 55,3 & 49,$2 ; 61,2$ & 374 & 44,7 & 38,$8 ; 50,8$ \\
\hline Análise dos dados & 395 & 47,2 & 41,$4 ; 53,1$ & 441 & 52,8 & 46,$9 ; 58,6$ \\
\hline Recomendação de ações/Tomada de decisões & 342 & 40,9 & 35,$2 ; 46,8$ & 494 & 59,1 & 53,$2 ; 64,8$ \\
\hline Execução de ações & 243 & 29,0 & 23,$9 ; 34,8$ & 593 & 71,0 & 65,$2 ; 76,1$ \\
\hline Divulgação das informações ou das ações executadas & 267 & 31,9 & 26,$6 ; 37,7$ & 569 & 68,1 & 62,$3 ; 73,4$ \\
\hline Controle de erros e inconsistências & 182 & 21,8 & 17,$4 ; 27,0$ & 654 & 78,2 & 73,$0 ; 82,6$ \\
\hline Identificação e busca do público & 287 & 34,3 & 28,$8 ; 40,2$ & 549 & 65,7 & 59,$8 ; 71,2$ \\
\hline Nenhuma das opções anteriores & 60 & 7,1 & 4,$6 ; 10,8$ & 776 & 92,9 & 89,$2 ; 95,4$ \\
\hline
\end{tabular}

a Peso, altura e/ou consumo alimentar.

ção básica; $48,9 \%$ com a secretaria municipal de assistência social (PBF); 39,6\% com a vigilância em saúde; $36,9 \%$ com as escolas.

Em relação à existência de espaço físico específico (sala individual ou uma estação de trabalho) para a gestão do SISVAN, $44,0 \%$ referiram possuir um espaço na SMS, 14,2\% fora da SMS e $39,5 \%$ não possuem espaço específico.

Considerando-se o uso do SISVAN, observase que um pouco mais da metade dos responsáveis pelo sistema coleta $(50,5 \%)$ e digita dados $(55,3 \%)$ no SISVAN Web, não analisa os dados $(52,8 \%)$ que o Sistema produz e não recomenda ações $(59,1 \%)$ que deveriam ser o resultado da análise dos dados no âmbito da gestão (Tabela 3 ).

Grande parte deles $(71,0 \%)$, não executa ações em decorrência destas informações, não divulga os resultados $(68,1 \%)$, não faz nenhum controle de erros ou de inconsistências $(78,2 \%)$ e não identifica e nem faz busca ativa $(65,7 \%)$ das crianças para o monitoramento do estado nutricional (Tabela 3).

Analisando o uso do SISVAN segundo a profissão, observa-se uma leve predominância dos nutricionistas em relação aos enfermeiros na digitação e análise dos dados, na execução de ações, na divulgação dos dados/ações e no controle de erros/inconsistências. Os enfermeiros têm maior atuação na coleta e na identificação/busca ativa. A recomendação de ações é realizada de forma igual, enquanto que a atuação dos técnicos de enfermagem se volta para as ações de coleta e digitação de dados, e dos técnicos de informática para a digitação.

A quase totalidade dos responsáveis pelo SISVAN coleta dados de peso e altura e somente $1 / 3$ deles registram o consumo alimentar. A maior parte da coleta de dados é realizada em atendi- mento individualizado ou no dia agendado para os beneficiários do PBF, seguida das chamadas nutricionais, visitas domiciliares e escolas. A grande maioria das crianças é simultaneamente atendida pelas ações de saúde da atenção básica do SUS e acompanhada pelo PBF (Tabela 4).

A maior parte das crianças diagnosticadas com alterações do estado nutricional (obesidade, sobrepeso, risco nutricional ou baixo peso) são encaminhadas para um acompanhamento específico, no momento da coleta de dados (Tabela $4)$.

Um pouco mais da metade dos responsáveis registra os dados concomitantemente no SISVAN e no módulo de gestão do PBF. Dentre os que registram os dados no SISVAN a periodicidade mais frequente é a mensal, seguida da não existência de uma periodicidade (Tabela 4).

A divulgação dos relatórios gerados pelo SISVAN é direcionada para os profissionais de saúde, seguido do secretário municipal de saúde e/ou prefeito e Conselho Municipal de Saúde (Tabela 4). O uso destes relatórios para menos de $40 \%$ dos entrevistados serve para subsidiar ações no campo da promoção da saúde, do cuidado e da prevenção.

Quanto ao conhecimento sobre a existência e origem do financiamento para a realização das atividades do SISVAN, 53,1\% dos entrevistados não souberam informar; $19,4 \%$ informaram a inexistência; $12,6 \%, 10,7 \%$ e $9,4 \%$ informaram que o financiamento é de origem federal, municipal e estadual, respectivamente.

Em relação aos documentos legais e técnicos utilizados pelos responsáveis pelo SISVAN, $68,6 \%$ registraram utilizar aqueles elaborados pela Coordenação Geral de Alimentação e Nutrição (CGAN) do Ministério da Saúde, 20,5\%, 
Tabela 4. Tipo de dados coletados, momento da coleta, o vínculo da criança tipo de sistema informatizado usado para o registro e periodicidade de registro no SISVAN Web - Minas Gerais, 2012.

\begin{tabular}{|c|c|c|c|}
\hline Variável & $\mathbf{N}$ & $\%$ & IC 95\% \\
\hline \multicolumn{4}{|l|}{ Tipo de dados coletados $(\mathrm{N}=836)$} \\
\hline Peso & 830 & 99,2 & 97,$0 ; 99,8$ \\
\hline Altura & 823 & 98,5 & 96,$0 ; 99,4$ \\
\hline Consumo alimentar & 302 & 36,1 & 30,$6 ; 41,9$ \\
\hline \multicolumn{4}{|l|}{ Momento da coleta de dados $(\mathrm{N}=836)$} \\
\hline Atendimento individualizado & 658 & 78,7 & 73,$4 ; 83,2$ \\
\hline No dia agendado para o Programa Bolsa Família & 542 & 64,9 & 58,$9 ; 70,4$ \\
\hline Chamada nutricional & 399 & 47,7 & 41,$7 ; 53,8$ \\
\hline Visita domiciliar & 391 & 46,8 & 40,$9 ; 52,7$ \\
\hline Nas escolas & 375 & 44,8 & 38,$9 ; 50,9$ \\
\hline Atendimento em grupo & 292 & 35,0 & 29,$5 ; 40,9$ \\
\hline Em dias de vacinação & 239 & 28,6 & 23,$5 ; 34,4$ \\
\hline Outro & 28 & 3,3 & 1,$7 ; 6,4$ \\
\hline \multicolumn{4}{|l|}{ Vínculo da criança com o serviço de saúde $(\mathrm{N}=836)$} \\
\hline Atenção Básica & 758 & 90,6 & 86,$5 ; 93,6$ \\
\hline Programa Bolsa Família & 674 & 80,6 & 75,$4 ; 85,0$ \\
\hline Programa Saúde na Escola & 239 & 28,6 & 23,$5 ; 34,4$ \\
\hline Programa Travessia ${ }^{\mathrm{a}}$ & 34 & 4,1 & 2,$3 ; 7,3$ \\
\hline Outro & 36 & 4,4 & 2,$5 ; 7,5$ \\
\hline \multicolumn{4}{|l|}{ Existência de encaminhamento $(\mathrm{N}=836)$} \\
\hline Encaminhamento dado à criança & 593 & 70,9 & 65,$3 ; 76,0$ \\
\hline Não sabe informar & 78 & 9,3 & 6,$4 ; 13,5$ \\
\hline \multicolumn{4}{|l|}{ Sistema informatizado de registro dos dados $(\mathrm{N}=836)$} \\
\hline Somente SISVAN Web & 212 & 25,4 & 20,$5 ; 31,0$ \\
\hline Somente Sistema de Gestão do Bolsa Família & 97 & 11,6 & 8,$3 ; 16,0$ \\
\hline Ambos os Sistemas & 505 & 60,4 & 54,$3 ; 66,1$ \\
\hline Outro & 13 & 1,5 & 0,$6 ; 3,9$ \\
\hline Não respondeu & 9 & 1,1 & 0,$4 ; 3,5$ \\
\hline \multicolumn{4}{|l|}{ Periodicidade do registro dos dados no SISVAN Web $(\mathrm{N}=717)$} \\
\hline Diariamente & 94 & 13,1 & 9,$3 ; 18,1$ \\
\hline Semanal & 112 & 15,7 & 11,$5 ; 21,0$ \\
\hline Quinzenal & 38 & 5,2 & 3,$0 ; 9,0$ \\
\hline Mensal & 214 & 29,9 & 24,$3 ; 36,1$ \\
\hline Semestral & 47 & 6,5 & 4,$0 ; 10,6$ \\
\hline Sem periodicidade específica & 181 & 25,2 & 20,$0 ; 31,4$ \\
\hline Não sabe informar & 31 & 4,4 & 2,$3 ; 8,0$ \\
\hline \multicolumn{4}{|l|}{ Divulgação dos Relatórios gerados pelo SISVAN Web $(\mathrm{N}=295)$} \\
\hline Profissionais de saúde & 264 & 89,5 & 81,$7 ; 94,2$ \\
\hline Secretário municipal de saúde/prefeito & 125 & 42,4 & 32,$8 ; 52,6$ \\
\hline Membros do Conselho Municipal de Saúde & 75 & 25,4 & 18,$0 ; 34,6$ \\
\hline População do município & 47 & 15,9 & 9,$9 ; 24,5$ \\
\hline Membros do Fórum/Conselho de Segurança Alimentar e Nutricional & 9 & 3,1 & 1,$0 ; 9,6$ \\
\hline Nenhuma das opções anteriores & 6 & 2,1 & 0,$5 ; 8,3$ \\
\hline \multicolumn{4}{|l|}{ Divulgação dos Relatórios gerados pelo SISVAN Web $(\mathrm{N}=480)$} \\
\hline Profissionais de saúde & 330 & 68,8 & 61,$0 ; 75,6$ \\
\hline Secretário municipal de saúde/prefeito & 292 & 61,0 & 52,$9 ; 68,4$ \\
\hline Membros do Conselho Municipal de Saúde & 299 & 62,3 & 54,$2 ; 69,8$ \\
\hline
\end{tabular}

${ }^{a}$ Programa do Estado de Minas Gerais voltado para localidades de menor Índice de Desenvolvimento Humano Municipal (IDH-M), destinados à população em situação de vulnerabilidade social.

$12,6 \%, 11,2 \%$ os elaborados pelo nível municipal, federal (de outros setores excluindo a CGAN) e estadual, respectivamente.
A maior parte dos responsáveis $(94,8 \%)$ reconhece que o SISVAN pode contribuir para o atendimento à saúde nutricional das crianças 
nos seguintes aspectos: monitoramento nutricional $(91,3 \%)$, diagnóstico precoce $(86,6 \%)$, direcionamento das ações da atenção básica $(85,8 \%)$, fortalecimento da área de alimentação e nutrição $(63,7 \%)$, diminuição de internações e uso de medicamentos $(61,0 \%)$, fortalecimento da área de Segurança Alimentar e Nutricional $(59,4 \%)$ e fortalecimento de parcerias $(42,1 \%)$.
As cinco principais dificuldades apontadas pelos responsáveis pelo SISVAN referiram-se a: insuficiência e falta de manutenção de equipamentos antropométricos (balanças e antropômetros), sobrecarga de trabalho do enfermeiro, internet lenta, falta de profissionais capacitados para a coleta, sobrecarga do trabalho do digitador, e falta de profissionais para a digitação dos dados (Tabela 5).

Tabela 5. Dificuldades na operacionalização e gestão dos SISVAN pelos responsáveis pelo Sistema - Minas Gerais, 2012.

\begin{tabular}{|c|c|c|c|c|}
\hline Dificuldades & $\mathbf{N}$ & $\%^{\mathrm{a}}$ & IC $95 \%$ & Posição ${ }^{b}$ \\
\hline \multicolumn{5}{|l|}{ Capacitações } \\
\hline Falta de profissionais capacitados para COLETA dos dados & 392 & 46,9 & 41,$0 ; 52,9$ & 4 \\
\hline Falta de profissionais capacitados para DIGITAÇÃO dos dados & 387 & 46,3 & 40,$4 ; 52,3$ & 6 \\
\hline Falta de profissionais capacitados para ANÁLISE dos dados & 325 & 38,8 & 33,$2 ; 44,8$ & 11 \\
\hline \multicolumn{5}{|l|}{ Número de profissionais } \\
\hline Quantidade insuficiente de nutricionistas & 368 & 44,0 & 38,$3 ; 49,9$ & 8 \\
\hline Quantidade insuficiente de profissionais, exceto nutricionistas & 189 & 22,6 & 18,$0 ; 28,1$ & 21 \\
\hline \multicolumn{5}{|l|}{ Sobrecarga de trabalho } \\
\hline Enfermeiro & 420 & 50,2 & 44,$3 ; 56,1$ & 2 \\
\hline Digitador & 389 & 46,5 & 40,$7 ; 52,4$ & 5 \\
\hline Nutricionista & 224 & 26,8 & 22,$0 ; 32,3$ & 19 \\
\hline \multicolumn{5}{|l|}{ Motivação e interação dos profissionais } \\
\hline Grande rotatividade de profissionais & 333 & 39,8 & 34,$1 ; 45,7$ & 10 \\
\hline Falta de interesse do profissional de saúde & 290 & 34,7 & 29,$3 ; 40,5$ & 15 \\
\hline Falta de interação entre os profissionais de saúde & 264 & 31,5 & 26,$3 ; 37,3$ & 17 \\
\hline Falta de interação entre os profissionais e os gestores municipais & 156 & 18,7 & 14,$4 ; 23,9$ & 24 \\
\hline \multicolumn{5}{|l|}{ Apoio de órgãos superiores } \\
\hline Falta de apoio da Secretaria Municipal de Saúde & 162 & 19,4 & 15,$1 ; 24,6$ & 23 \\
\hline Falta de apoio da Gerência Regional de Saúde/Secretaria Estadual de Saúde & 128 & 15,3 & 11,$5 ; 20,1$ & 26 \\
\hline Falta de apoio do Ministério da Saúde & 97 & 11,6 & 8,$3 ; 16,0$ & 29 \\
\hline \multicolumn{5}{|l|}{ Equipamentos } \\
\hline Equipamentos antropométricos insuficientes ou sem manutenção & 451 & 54,0 & 48,$0 ; 59,9$ & 1 \\
\hline Computadores insuficientes ou sem manutenção & 340 & 40,7 & 34,$9 ; 46,7$ & 9 \\
\hline \multicolumn{5}{|l|}{ Acesso à internet } \\
\hline Internet lenta & 399 & 47,7 & 41,$8 ; 53,7$ & 3 \\
\hline SISVAN Web fora do ar & 384 & 46,0 & 40,$1 ; 51,9$ & 7 \\
\hline Faltam computadores com internet para digitar os dados coletados & 188 & 22,4 & 17,$8 ; 27,9$ & 22 \\
\hline Falta acesso à internet & 114 & 13,7 & 10,$1 ; 18,3$ & 27 \\
\hline \multicolumn{5}{|l|}{ Digitação dos dados } \\
\hline Dificuldades para coleta dos dados de cadastro & 309 & 36,9 & 31,$4 ; 42,9$ & 13 \\
\hline $\begin{array}{l}\text { Priorização da coleta dos dados do Bolsa Família em detrimento dos dados } \\
\text { do SISVAN }\end{array}$ & 299 & 35,8 & 30,$3 ; 41,7$ & 14 \\
\hline Dúvidas ao digitar os dados no SISVAN Web & 191 & 22,8 & 18,$2 ; 28,3$ & 20 \\
\hline Os dados coletados não são digitados no SISVAN Web & 135 & 16,1 & 12,$2 ; 21,0$ & 25 \\
\hline \multicolumn{5}{|l|}{ Financiamento } \\
\hline $\begin{array}{l}\text { Falta recurso financeiro para o desenvolvimento e execução de ações/ } \\
\text { intervenções nutricionais }\end{array}$ & 309 & 37,0 & 31,$4 ; 43,0$ & 12 \\
\hline Falta recurso financeiro para operacionalização do SISVAN & 266 & 31,8 & 26,$4 ; 37,6$ & 16 \\
\hline Dificuldades para executar (utilizar) os recursos financeiros & 113 & 13,5 & 9,$8 ; 18,2$ & 28 \\
\hline \multicolumn{5}{|l|}{ Território } \\
\hline Grande extensão e dificuldade de acesso da zona rural & 256 & 30,6 & 25,$3 ; 36,5$ & 18 \\
\hline Dificuldade de acesso do PSF/ESF/PACS à população adscrita & 68 & 8,1 & 5,$4 ; 12,0$ & 30 \\
\hline
\end{tabular}

${ }^{a} \mathrm{O}$ total equivale a 836 . ${ }^{\text {b }}$ Foi feito um ranking em que o número 1 foi a dificuldade que possui a maior \%, ou seja, foi a dificuldade apontada pelo maior número de responsáveis pelo SISVAN. 
Os entrevistados atribuíram uma nota à utilidade do SISVAN para a melhoria do estado nutricional de crianças, onde as notas zero e dez expressaram respectivamente nenhuma e muita utilidade. Observa-se que 35,9\% dos responsáveis deram nota igual ou inferior a $6 ; 35,5 \%$ entre 7 e 8 ; e, $26,8 \%$ entre 9 e 10 ; a média, a mediana e a moda corresponderam a 7,12; 7 e 8 , respectivamente.

\section{Discussão}

O perfil dos responsáveis pelo SISVAN identificado pelo estudo é predominantemente feminino e jovem, não difere daquele encontrado no Brasil para os profissionais da área de saúde em geral. A participação da mulher no mercado de trabalho é crescente, principalmente nas faixas etárias mais jovens e hoje elas representam $70 \%$ de toda força de trabalho no setor saúde ${ }^{21,22}$. Ademais, a nutrição e a enfermagem são profissões consideradas femininas.

A predominância do nutricionista, depois do enfermeiro, como responsável pelo Sistema, em parte, expressam a recomendação da Portaria $\mathrm{n}^{\circ} 2.246 / 2004$, que propõe o nutricionista para a coordenação do SISVAN ${ }^{13,23}$, e são coincidentes com os resultados encontrados por Romeiro ${ }^{13}$ no âmbito nacional.

Destaca-se o fato dos nutricionistas e enfermeiros, profissionais centrais para o desenvolvimento das atividades do SISVAN, possuírem menor estabilidade no vínculo trabalhista do que os profissionais com formação técnica. Medeiros et. $\mathrm{a}^{24}$ mostram que o vínculo tem sido valorizado pelos médicos e enfermeiros das equipes da ESF por representar garantia ao profissional. Atualmente, os gestores da saúde vêm enfrentando dificuldades com a fixação de profissionais em municípios de pequeno porte e muitas prefeituras não têm condições para realizar concursos públicos $^{12,24}$. Além dos vínculos não estáveis, as grandes diferenças salariais entre os municípios podem induzir alta rotatividade na equipe $\mathrm{e}^{24}$, uma das dificuldades encontrada neste estudo (classificada na $10^{\text {a }}$ posição) e por Romeiro ${ }^{13}$.

Destaca-se que a maior parte dos responsáveis pelo SISVAN informou executar prioritariamente atividades relacionadas a coleta e digitação dos dados que, segundo as publicações oficiais ${ }^{9,25}$, podem ser realizadas por profissionais capacitados para estas atividades, cabendo ao responsável pelo SISVAN apenas orientar e acompanhar sua realização.
A ausência da análise dos dados e da implementação de ações dela decorrentes são atividades que demonstram a subutilização das informações do SISVAN no âmbito da gestão municipal da atenção básica do SUS. Assim como a ausência ou o pouco controle sobre os erros e inconsistências na coleta e digitação dos dados, também identificados no presente estudo. Batista-Filho e Rissin ${ }^{14}$, analisando a realidade nacional identificaram que a baixa qualidade dos dados e a dificuldade em analisá-los e interpretá-los não motivam intervenções em prol da melhoria do estado nutricional. A utilização das informações rotineiramente produzidas nos serviços de saúde pelos próprios profissionais de saúde contribui para melhorar sua qualidade e potencializar o seu uso pelos gestores ${ }^{10,14,25}$.

Alguns municípios apontaram como limitador a insuficiência de nutricionistas na operacionalização das atividades do SISVAN. A formação acadêmica deste profissional possibilita a interpretação do estado nutricional individual e coletivo, mostrando uma competência técnica mais especializada para o uso do SISVAN com maior potencialidade $^{13}$, que contribui para fechar o ciclo desde a coleta de dados até a execução de ações, passando pela divulgação e análise de erros e inconsistências.

Outro facilitador para o uso em potencial do SISVAN parece ser o envolvimento dos seus responsáveis em outras atividades no âmbito da alimentação e nutrição, atenção básica, assistência social e educação. Neste contexto, Arruda ${ }^{26}$ identifica que a implantação do SISVAN de forma isolada dentro do setor saúde é um problema para a sua execução.

Em contraposição, a sobrecarga de trabalho foi identificada no estudo como importante dificuldade no desenvolvimento das atividades do SISVAN, particularmente em relação ao enfermeiro, este profissional encontra-se à frente da coordenação da atenção básica e com acúmulo de atividades que extrapolam o âmbito da alimentação e nutrição.

Como esperado as capacitações foram realizadas em sua quase totalidade pelo nível estadual que priorizou em seu conteúdo a coleta e, após a criação do SISVAN Web, a digitação dos dados evidenciando a necessidade de ajuste do conteúdo ministrado de modo a despertar os profissionais para uma atuação que possibilite o uso das informações geradas pelo SISVAN como um instrumento para tomada de decisão, capaz de implementar as diretrizes da PNAN. Tal conclusão é compatível com a Matriz de Ações de Alimenta- 
ção e Nutrição na Atenção Básica de Saúde ${ }^{5}$, que destaca a necessidade de incluir conteúdos sobre a gestão no âmbito das ações de alimentação e nutrição, incluindo a análise de dados e o uso das informações para ação e para a gestão. Ademais, as capacitações devem fazer parte de um processo de atualização permanente e contínuo que acompanhe as mudanças sociopolíticas, demográficas e de morbimortalidade.

Os documentos utilizados pela maioria dos responsáveis pelo SISVAN para orientar suas ações foram elaborados pela CGAN/MS, sendo normativos e focados na antropometria e no cuidado com a criança ${ }^{27}$. Apesar de o objetivo do estudo não ter sido avaliar o conteúdo dos documentos oficiais, observa-se a necessidade de que estes também atribuam maior ênfase ao conteúdo da gestão, ampliando e consolidando o SISVAN como instrumento de informação útil para a tomada de decisões estratégias de alimentação e nutrição da atenção básica do SUS ${ }^{27}$.

A formação desses profissionais em gestão possibilita melhor utilização dos recursos disponíveis para o campo da alimentação e nutrição, abrindo uma janela de oportunidades para o profissional responsável pelo SISVAN resolver as questões de infraestrutura e fortalecer e qualificar o cuidado nutricional no âmbito de sua atuação. A organização dos sistemas públicos de saúde, planejamento, orçamento, financiamento e recursos humanos, são ações da gestão públi$\mathrm{ca}$, as quais os profissionais de saúde precisam se apropriar e, de fato, atuar na adequação ou implementação dos serviços necessários à atenção à saúde em seus âmbitos de atuação ${ }^{28}$.

Destaca-se que na SES-MG, o SISVAN, diferente da maioria dos municípios de Minas Gerais e do nível federal, não está vinculado formalmente à atenção básica como preconizado pela $\mathrm{PNAN}^{3}$, que propõe a atenção básica como ordenadora da atenção nutricional no SUS, sugerindo certa fragilidade na sua institucionalização. Atualmente, a CGAN organiza uma rede composta por profissionais envolvidos na implementação de ações de alimentação e nutrição em diferentes esferas de governo e áreas (Rede de Nutrição do Sistema Único de Saúde - RedeNutri), e estimula uma estrutura padronizada de fluxo de informações de alimentação e nutrição com o nível federal, via SISVAN Web ${ }^{15,17}$.

Os resultados do presente estudo sugerem a ampliação das parcerias para o desenvolvimento do SISVAN, considerando que as ações de alimentação e nutrição possuem determinação social, natureza interdisciplinar e intersetorial ${ }^{3}$.
Romeiro $^{13}$ identificou que apenas 33\% dos municípios do Brasil e 75\% dos estados estabeleceram parceria em 2006. Evidencia-se assim o desafio da participação multisetorial, tendo em vista os conflitos de interesses das diferentes instâncias envolvidas e ausência de modelos realistas de coordenação ${ }^{26}$.

O pressuposto era de que havia uma prioridade para a digitação dos dados de antropometria, no módulo de gestão do PBF, uma vez que o seu preenchimento está condicionado ao recebimento de recursos financeiros para o município. Porém, diferente do esperado, os resultados mostram que os profissionais utilizam ambos os sistemas, o que é positivo, pois entende-se que as crianças usuárias do SUS e aquelas beneficiárias do PBF estão tendo seu perfil antropométrico acompanhado. Em estudo na Superintendência Regional de Saúde de Belo Horizonte (SRS-BH) analisando crianças menores de 2 anos, Ferreira et. al encontraram maior acompanhamento pelo SISVAN-Web nos anos de 2009 e 2011, e pelo módulo de gestão do PBF nos anos de 2008 e 2010.

No que tange a coleta de dados do consumo alimentar, apesar de se observar uma baixa adesão pelos municípios, considera-se um resultado positivo e que deve ser estimulada para os demais municípios, tendo em vista que o seu uso potencializa e amplia a utilização do SISVAN, para além da antropometria. Ferreira et al. ${ }^{29}$ encontraram uma porcentagem menor dos municípios que digitam dados de consumo alimentar na SRS-BH. É importante ressaltar que, em Minas Gerais, o processo de implantação da Estratégia Nacional para Alimentação Complementar Saudável (ENPACS), iniciado em 2009, estimulou a coleta desses dados em virtude de a avaliação do consumo alimentar de crianças menores de 2 anos ser uma etapa do seu monitoramento.

Os responsáveis pelo SISVAN, predominantemente, informaram que encaminham as crianças identificadas com algum distúrbio nutricional para atendimento diferenciado. O encaminhamento é uma orientação dos documentos oficiais $^{9}$ e proporciona utilidade ao SISVAN no nível local, tornando possível o uso rápido da informação. Ressalta-se que cerca de $1 / 5$ dos entrevistados informaram não encaminhar as crianças para outro tipo de atendimento, o que pode ser positivo caso o problema diagnosticado tenha sido resolvido no mesmo atendimento que o identificou, ou negativo se for devido à ausência de um serviço de referência.

Destaque-se que poucos referiram utilizar os relatórios para este fim, divergindo da orientação 
do $\mathrm{MS}^{3}$, apesar de grande parte dos responsáveis pelo SISVAN considerar importante o seu uso para orientar as ações, sejam elas de promoção, prevenção ou cuidado ou para divulgar as informações do estado nutricional e do consumo alimentar para outros profissionais, setores ou para a população. A não utilização das informações foi identificada em outros momentos da trajetória nacional do SISVAN ${ }^{13-15,29}$ e pode estar relacionada especialmente à não apropriação do exercício de planejamento e gestão das ações no campo da nutrição, ou com a falta de confiança na qualidade dos dados. Cabe destacar que apesar de grande parte dos profissionais acreditar na importância do monitoramento do estado nutricional das crianças, poucos utilizam suas informações.

Este achado sugere que há um distanciamento na compreensão entre o que seja monitoramento do estado nutricional e utilização do SISVAN, que pode ser decorrente da interpretação dada aos documentos oficiais e capacitação inadequada para o uso da informação para a ação. Por exemplo, a portaria n. ${ }^{\circ} 2.246$ define um setor para o suporte técnico do sistema informacional para a entrada e o processamento de dados, e outro setor para o suporte técnico das atividades do SISVAN $^{15,23}$.

O uso das informações para a ação tem sido um desafio e atualmente o MS tem implementado o e-SUS Atenção Básica (Sistema informatizado para a Atenção Básica) como uma estratégia de integração dos diversos SIS no âmbito da atenção básica do SUS, entendendo que a qualificação da gestão da informação é fundamental para ampliar a qualidade no atendimento à população.

\section{Colaboradores}

MD Rolim foi responsável pelo estudo, desenho do estudo, elaboração do questionário, coordenação e realização do trabalho de campo, realização da análises e redação do manuscrito. SML Lima participou desenho do estudo, revisão do questionário, coordenação do trabalho de campo e redação do manuscrito. DC Barros participou do desenho do estudo, revisão do questionário e redação do manuscrito. CLT Andrade participou do desenho do estudo, desenho amostral, revisão do questionário e redação do manuscrito.
A principal limitação encontrada pode ser apontada para uso de questionário eletrônico para coleta das informações junto aos sujeitos do estudo que exigiu um maior empenho dos pesquisadores no contato com os municípios, para manter o tamanho da amostra. Houve dificuldades dos sujeitos em retornar as respostas e para salvar as informações online, obrigando o respondente a fazê-lo novamente.

Concluindo, a grande maioria dos responsáveis pelo SISVAN não executam as atividades que expressariam o seu uso como base para a tomada de decisão e, muitos, não dão a devida importância ao uso de suas informações para orientar a gestão municipal, apesar de informarem que utilizam os relatórios para subsidiar ações de promoção da saúde, prevenção e cuidado e reconhecerem o seu potencial para a melhoria do estado nutricional. A execução do SISVAN deve estar vinculada às outras atividades de alimentação e nutrição e as capacitações, assim como os materiais oficiais devem focar na gestão das ações e serviços de nutrição, fortalecendo a realização do SISVAN para o planejamento e execução de ações. Ao que parece, o preenchimento dos dados no sistema não é visto como uma atividade propulsora da vigilância nutricional e nem geradora de dados fundamentais para os tomadores de decisão. Portanto, as informações geradas pelo SISVAN não têm sido utilizadas em seu potencial para o planejamento, gestão e avaliação de ações de alimentação e nutrição no âmbito da atenção básica do SUS em Minas Gerais e, consequentemente, não contribuindo para implementação da PNAN. 


\section{Referências}

1. Batista-Filho M, Rissin A. Transição nutricional no Brasil: tendências regionais e temporais. Cad Saude Publica 2003; 19(1):181-191.

2. Instituto Brasileiro de Geografia e Estatística (IBGE). Pesquisa de Orçamentos Familiares 2008/2009 - Antropometria e estado nutricional de crianças, adolescentes $e$ adultos no Brasil. Rio de Janeiro: IBGE; 2010.

3. Brasil. Portaria no ${ }^{\circ} .715$ de 17 de novembro de 2011. Atualiza a Política Nacional de Alimentação e Nutrição. Diário Oficial da União 2011; 17 nov.

4. Schmidt MI, Duncan BB, Azevedo e Silva G, Menezes AM, Monteiro CA, Barreto SM, Chor D, Menezes PR. Doenças crônicas não transmissíveis no Brasil: carga e desafios atuais. Lancet 2011; 4(9):61-74.

5. Brasil. Ministério da Saúde (MS). Matriz de ações de alimentação e nutrição na atenção básica de saúde. Brasília: MS; 2009. (Série A - Normas e manuais técnicos).

6. Recine E, Vasconcellos AB. Políticas nacionais e o campo da alimentação e nutrição em saúde coletiva: cenário atual. Cien Saude Colet 2011; 16(1):73-79.

7. Brasil. Portaria $n^{\circ} 710$ de 10 de junho de 1999. Aprova a Política Nacional de Alimentação e Nutrição. Diário Oficial da União 1999; 10 jun.

8. Brasil. Ministério da Saúde (MS). Indicadores de Vigilância Alimentar e Nutricional - Brasil 2006. Brasília: MS; 2009.

9. Brasil. Ministério da Saúde (MS). Vigilância alimentar e nutricional - SISVAN: orientações básicas para a coleta, o processamento, a análise de dados e a informação em serviços de saúde. Brasília: MS; 2004. (Série A - Normas e manuais técnicos).

10. Sistema de Informação da Atenção Básica [Internet]. Rio de Janeiro: DATASUS -[acessado 2011 nov 17]. Disponível em: http://siab.datasus.gov.br/.

11. Coutinho JG, Cardoso AJC, Toral N, Silva ACF, Ubarana JA, Aquino KKNC, Nilson EAF, Fagundes A, Vasconcellos AB. A organização da Vigilância Alimentar e Nutricional no Sistema Único de Saúde: histórico e desafios atuais. Rev. Bras. Epidemiol. 2009; 12(4):688-699.

12. Medeiros KR, Machado HOP, Albuquerque PC, Gurgel Junior GD. O Sistema de Informação em Saúde como instrumento da política de recursos humanos: um mecanismo importante na detecção das necessidades da força de trabalho para o SUS. Cien Saude Colet 2005; 10(2):433-440.

13. Romeiro AAF. Avaliação do Sistema de Vigilância Alimentar e Nutricional - SISVAN, no Brasil. Brasília [dissertação]. Brasília: Universidade de Brasília; 2006.

14. Batista-Filho M, Rissin A. Vigilância alimentar e nutricional: antecedentes, objetivos e modalidades. Cad Saude Publica 1993; 9(1):99-105.

15. Vasconcellos ABPA. As Ações da Política Nacional de Alimentação e Nutrição na rede de atenção básica: uma análise dos anos de 2008 e 2009 [dissertação]. Rio de Janeiro: Fiocruz; 2011.

16. Instituto Brasileiro de Geografia e Estatística (IBGE). Censo Demográfico 2010. Rio de Janeiro. [acessado 2011 maio 31]. Disponível em: http://www.ibge.gov. $\mathrm{br} /$.

17. Coordenação Geral de Alimentação e Nutrição [Internet]. Brasília. [acessado 2011 maio 31]. Disponível em: http://www.saude.gov.br/nutrição/.
18. Cochran WG. Sampling techniques. $3^{\text {rd }}$ ed. New York: Ed. John Wiley \& Sons; 1977.

19. Avaliação da estrutura organizacional e do processo de implantação do SISVAN WEB no âmbito da Atenção Básica no estado de Minas Gerais, Brasil. [Internet]. Ouro Preto (MG). [acessado 2011 jul 26]. Disponível em: http://spreadsheets.google.com/viewform?form key $=$ dHJGYkNzOXVLcWZ4djUzY25xS3pEVnc6MQ/.

20. Brasil. Ministério da Saúde (MS). Conselho Nacional de Saúde. Resolução no 196 de 10 de outubro de 1996. Diretrizes e Normas Regulamentadoras de Pesquisas Envolvendo Seres Humanos. Diário Oficial da União 1996; 16 out.

21. Machado MH, Wermelinger M, Tavares MFL, Moysés NMN, Teixeira M, Oliveira ES. Análise da Força de Trabalho do. Setor Saúde no Brasil: focalizando a feminização. Rio de Janeiro: Fiocruz; 2006.

22. Oba MV, Kinouchi FL, Scandiuzzi RJ, Soares DW, Brandão ML. O ser mulher mediante as representações sociais dos profissionais de saúde. J Health Sci Inst 2012; 30(4):343-348.

23. Brasil. Portaria $n^{\circ} 2.246 / G M$ de 18 de outubro de 2004 . Institui e divulga orientações básicas para a implementação das Ações de Vigilância Alimentar e Nutricional, no âmbito das ações básicas de saúde do Sistema Único de Saúde - SUS, em todo o território nacional. Diário Oficial da União 2004; 18 out.

24. Medeiros CRG, Junqueira AGW, Schwingel G, Carreno I, Jungles LAP, Saldanha OMFL. A rotatividade de enfermeiros e médicos: um impasse na implementação da Estratégia de Saúde da Família. Cien Saude Colet 2010; 15(1):1521-1531.

25. Brasil. Ministério da Saúde (MS). Protocolos do Sistema de Vigilância Alimentar e Nutricional - SISVAN na assistência à saúde. Brasília: MS; 2008. (Série B - textos básicos de saúde).

26. Arruda BKG. Sistema de vigilância alimentar e nutricional. Frustrações, desafios e perspectivas. Cad Saude Publica 1992; 8(1):96-101.

27. Costa KMRP. Análise das estratégias de informação e educação sobre alimentação e nutrição produzidas no âmbito da Política Nacional de Alimentação e Nutrição, no período de 1999 a 2010 [tese]. Rio de Janeiro: Fiocruz; 2010.

28. Brasil. Conselho Nacional de Secretários de Saúde (CONASS). A Gestão Administrativa e Financeira no SUS/ Conselho Nacional de Secretários de Saúde. Brasília: CONASS; 2011.

29. Ferreira CS, Cherchiglia ML, César CC. O Sistema de Vigilância Alimentar e Nutricional como instrumento de monitoramento da Estratégia Nacional para Alimentação Complementar Saudável. Rev. Bras. Saude Mater. Infant. 2013; 13(2):167-177.

Artigo apresentado em 11/02/2015

Aprovado em 11/04/2015

Versão final apresentada em 13/04/2015 